\title{
REGULARITY OF LANGUAGES DEFINED BY FORMAL SERIES WITH ISOLATED CUT POINT*
}

\author{
Alberto Bertoni ${ }^{1}$, Maria Paola Bianchi ${ }^{1}$ \\ and Flavio D'Alessandro ${ }^{2}$
}

\begin{abstract}
Let $L_{\varphi, \lambda}=\left\{\omega \in \Sigma^{*} \mid \varphi(\omega)>\lambda\right\}$ be the language recognized by a formal series $\varphi: \Sigma^{*} \rightarrow \mathbb{R}$ with isolated cut point $\lambda$. We provide new conditions that guarantee the regularity of the language $L_{\varphi, \lambda}$ in the case that $\varphi$ is rational or $\varphi$ is a Hadamard quotient of rational series. Moreover the decidability property of such conditions is investigated.
\end{abstract}

Mathematics Subject Classification. 68Q45, 68Q70.

\section{INTRODUCTION}

In this paper, we investigate some problems concerning formal power series $\varphi: \Sigma^{*} \rightarrow \mathbb{R}$ in noncommutative variables with coefficients in the field of real numbers. Formal power series in noncommutative variables have a deep impact on several areas of mathematics and computer science. Indeed, they provide a powerful tool to describe the behavior of various models of acceptors of formal languages such as, for instance, grammars [10], probabilistic automata [11], and quantum automata [5] (see also [2,19]).

\footnotetext{
Keywords and phrases. Formal power series, Hadamard quotient, regular languages.

* The authors wish to thank Christian Choffrut for his help in improving a previous version of this paper.

1 Dipartimento di Informatica, Università degli Studi di Milano Via Comelico 39, 20135 Milano, Italy. bertoni@di.unimi.it; bianchi@di.unimi.it

2 Dipartimento di Matematica, Università di Roma "La Sapienza" Piazzale Aldo Moro 2, 00185 Roma, Italy. dalessan@mat.uniroma1.it
} 
Given a number $\lambda \in \mathbb{R}$ and a series $\varphi: \Sigma^{*} \rightarrow \mathbb{R}$, we can associate to $\varphi$ and $\lambda$ the language $L_{\varphi, \lambda}=\left\{\omega \in \Sigma^{*} \mid \varphi(\omega)>\lambda\right\}$. The value $\lambda$ is said to be isolated for $\varphi$ if $\inf _{\omega \in \Sigma^{*}}|\varphi(\omega)-\lambda|>0$.

In this theoretical frame, a classical line of investigation concerns the study of the so-called regularity conditions, that is, conditions on $\varphi$ and $\lambda$ which guarantee that $L_{\varphi, \lambda}$ is a regular language. It is worth mentioning that a celebrated theorem by Rabin [18] shows the importance of the notion of isolated cut point as a regularity condition for languages accepted by finite probabilistic automata.

In this paper the first result we prove concerns a regularity condition for the class $\mathbb{R}^{\text {rat }}\left\langle\left\langle\Sigma^{*}\right\rangle\right\rangle$ of rational power series. More precisely, we prove that, if $\varphi \in \mathbb{R}^{\text {rat }}\left\langle\left\langle\Sigma^{*}\right\rangle\right\rangle$ is bounded and $\lambda$ is isolated, then $L_{\varphi, \lambda}$ is a regular language.

We then investigate regularity conditions for the Hadamard quotient of series of $\mathbb{R}^{\text {rat }}\left\langle\left\langle\Sigma^{*}\right\rangle\right\rangle$. It is well known that the class of rational series is closed with respect to the Hadamard product of series, while it is not closed with respect to the Hadamard quotient. In the univariate case, a remarkable result is due to Van Der Poorten [21]: if the Hadamard quotient of two rational functions is a Taylor series with integer coefficients, then it is a rational function.

Another reason that makes the Hadamard quotient an interesting operation is that the behavior defined by a two-way probabilistic automaton can be expressed as the Hadamard quotient (generally non-rational) of two rational series [1].

The second result of this paper is the following. Let $\varphi, \psi$ be two bounded rational power series of $\mathbb{R}^{\text {rat }}\left\langle\left\langle\Sigma^{*}\right\rangle\right\rangle$ and let $\xi$ be the Hadamard quotient of $\varphi$ and $\psi$. Under the assumption that 0 is isolated for $\psi$ and $\lambda$ is isolated for $\xi$, we have that $L_{\xi, \lambda}$ is regular. Moreover, we show by counterexamples that all hypotheses are necessary, even in the univariate case. The proof of these results is based upon an argument of Diophantine approximation.

Finally, we show that the boundedness problem for rational power series of $\mathbb{R}^{\text {rat }}\left\langle\left\langle\Sigma^{*}\right\rangle\right\rangle$ and the regularity problem for languages defined by rational power series of $\mathbb{R}^{\text {rat }}\left\langle\left\langle\Sigma^{*}\right\rangle\right\rangle$, with isolated cut point, are undecidable.

\section{Preliminaries}

Let $\mathbb{R}$ be the field of real numbers. For every $r \in \mathbb{R},|r|$ indicates the absolute value of $r$. We denote by $\mathbb{R}^{n \times m}$ the set of $n \times m$ matrices with real entries. For every vector $\mathbf{v}=\left(v_{1}, \ldots, v_{n}\right) \in \mathbb{R}^{1 \times n}$, we denote by

$$
\|\mathbf{v}\|_{2}=\sqrt{v_{1}^{2}+\cdots+v_{n}^{2}}, \quad\|\mathbf{v}\|_{\infty}=\max _{1 \leq i \leq n}\left|v_{i}\right|
$$

the Euclidean norm and the maximum norm of $\mathbf{v}$, respectively. Note that for every $\mathbf{v} \in \mathbb{R}^{1 \times n}$ we have

$$
\|\mathbf{v}\|_{\infty} \leq\|\mathbf{v}\|_{2} \leq n\|\mathbf{v}\|_{\infty}
$$


For matrices $A \in \mathbb{R}^{n \times m}$ and $B \in \mathbb{R}^{p \times q}$, their direct sum is the $(n+p) \times(m+q)$ matrix defined as

$$
A \oplus B=\left(\begin{array}{cc}
A & \mathbf{0}^{n \times q} \\
\mathbf{0}^{p \times m} & B
\end{array}\right)
$$

where $\mathbf{0}^{h \times k}$ denotes the $h \times k$ zero matrix. With a slight abuse of notation, we define the direct sum of two vectors $\pi \in \mathbb{R}^{1 \times n}$ and $\xi \in \mathbb{R}^{1 \times m}$, as the $1 \times(n+m)$ vector $\pi \oplus \xi=\left(\pi_{1}, \ldots, \pi_{n}, \xi_{1}, \ldots, \xi_{m}\right)$.

Given a function $f: D \rightarrow \mathbb{R}$, the set of accumulation points of $f$, denoted $\operatorname{Lim}\{f(x) \mid x \in D\}$, is the set:

$$
\left\{\ell \in \mathbb{R} \mid \forall \varepsilon \in \mathbb{R} \exists x_{s} \in D \text { such that } 0<\left|f\left(x_{s}\right)-\ell\right|<\varepsilon\right\} .
$$

Given $r \in \mathbb{R}$, the best integer approximation of $r$ is $q(r)=\arg \min _{z \in \mathbb{Z}}|z-r|$ and the quantization error is $\{r\}=|r-q(r)|$. Given two sequences $f(n)$ and $g(n)$, we set $f(n) \sim g(n)$ iff $\lim _{n \rightarrow \infty} f(n) / g(n)=1$. The following lemma is immediate.

Lemma 2.1. Let $f(n)$ be a sequence satisfying $|f(n)| \leq \frac{1}{2}$ for every $n$. If $\lim _{n \rightarrow \infty}$ $|\sin (\pi f(n))|=0$, then $|\sin (\pi f(n))| \sim \pi|\{f(n)\}|$.

Let $\Sigma^{*}$ be the free monoid generated by the finite alphabet $\Sigma$; we denote by $\epsilon$ the empty word. Given a word $\omega \in \Sigma^{*}$, we write $|\omega|$ for the length of $\omega$. Given an alphabet $\Sigma$, a word $\omega \in \Sigma^{*}$ and a symbol $\sigma \in \Sigma$, we denote by $\#_{\sigma}(\omega)$ the number of occurrences of the symbol $\sigma$ in $\omega$. Given an alphabet $\Gamma \subseteq \Sigma$, we denote by $P_{\Gamma}(\omega)$ the projection of $\omega$ on the alphabet $\Gamma$, i.e. for $\omega=\sigma_{1} \cdots \sigma_{n}$ it holds $P_{\Gamma}(\omega)=P_{\Gamma}\left(\sigma_{1}\right) \cdots P_{\Gamma}\left(\sigma_{n}\right)$ and for each $\sigma \in \Gamma$ it holds $P_{\Gamma}(\sigma)=\sigma$, while for $\sigma \in \Sigma-\Gamma$ it holds $P_{\Gamma}(\sigma)=\epsilon$.

A formal power series (in noncommutative variables) with coefficients in the field $\mathbb{R}$ is any function $\varphi: \Sigma^{*} \rightarrow \mathbb{R}$, usually expressed by the formal sum $\varphi=\sum_{\omega \in \Sigma^{*}} \varphi(\omega) \omega$. The class of all formal power series $\varphi: \Sigma^{*} \rightarrow \mathbb{R}$ is written as $\mathbb{R}\left\langle\left\langle\Sigma^{*}\right\rangle\right\rangle$. The support of $\varphi$ is the language $\operatorname{supp}(\varphi)=\left\{\omega \in \Sigma^{*} \mid \varphi(\omega) \neq 0\right\}$. A polynomial is a series with finite support, and the class of polynomials is denoted by $\mathbb{R}\left\langle\Sigma^{*}\right\rangle$. For the sake of simplicity, for any $a \in \mathbb{R}$, we write $a$ for the polynomial $a \epsilon$. A ring structure is defined on $\mathbb{R}\left\langle\left\langle\Sigma^{*}\right\rangle\right\rangle$ by introducing the operations of sum and Cauchy product:

Sum: $(\varphi+\psi)(\omega)=\varphi(\omega)+\psi(\omega)$.

Cauchy product: $(\varphi \cdot \psi)(\omega)=\sum_{\substack{x, y \in \Sigma^{*} \\ x y=\omega}} \varphi(x) \psi(y)$.

A series $\varphi$ is proper if $\varphi(\epsilon)=0$; in this case, the star operation can be defined as

$$
\varphi^{*}=\sum_{i \geq 0} \varphi^{i}, \text { where } \varphi^{0}=1 \text { and } \varphi^{i}=\varphi \cdot \varphi^{i-1}, \text { for } i>0 .
$$


An important subclass of $\mathbb{R}\left\langle\left\langle\Sigma^{*}\right\rangle\right\rangle$ is the class of rational series:

Definition 2.2. The class $\mathbb{R}^{\text {rat }}\left\langle\left\langle\Sigma^{*}\right\rangle\right\rangle$ of rational power series is the smallest subclass of $\mathbb{R}\left\langle\left\langle\Sigma^{*}\right\rangle\right\rangle$ containing $\mathbb{R}\left\langle\Sigma^{*}\right\rangle$ that is closed under the rational operations of sum, Cauchy product and star of proper series.

A triple $(\nu, \mu, \eta)$, with $\nu, \eta \in \mathbb{R}^{1 \times m}$ and, for every $\sigma \in \Sigma, \mu(\sigma) \in \mathbb{R}^{m \times m}$ is a linear representation of dimension $m$ of a series $\varphi: \Sigma^{*} \rightarrow \mathbb{R}$, iff for any word $\omega=\sigma_{1} \sigma_{2} \cdots \sigma_{n} \in \Sigma^{*}$ we get

$$
\varphi(\omega)=\nu \mu(\omega) \eta^{T}=\nu\left(\prod_{i=1}^{n} \mu\left(\sigma_{i}\right)\right) \eta^{T} .
$$

A series that admits a linear representation is called recognizable. In [20], Schützenberger proved that a formal power series is rational iff it is recognizable. This result can be seen as an extension of Kleene's theorem.

One important application of rational formal power series is the description of the behavior of one-way probabilistic and quantum finite state automata.

Example 2.3. A probabilistic automaton [17, 18] (1pfa, for short) on $\Sigma$ with $m$ states is represented by $A=(\nu, \mu, \eta)$, where $\nu$ is a $1 \times m$ stochastic vector (probability distribution of initial states), $\mu(\sigma)$ is an $m \times m$ stochastic matrix (transition matrix), for every $\sigma \in \Sigma$, and $\eta \in\{0,1\}^{m}$ is the characteristic vector of final states; $A$ is the linear representation of the formal series $\mathcal{P}_{A}: \Sigma^{*} \rightarrow[0,1]$, where $\mathcal{P}_{A}(\omega)$ is the probability of accepting the word $\omega$.

Given a formal power series $\varphi$ and a real $\lambda$, the language $L_{\varphi, \lambda}$ defined by $\varphi$ with cut-point $\lambda$ is written as $L_{\varphi, \lambda}=\left\{\omega \in \Sigma^{*} \mid \varphi(\omega)>\lambda\right\}$. The cut-point is said to be isolated if there exists a positive $\delta$ such that $|\varphi(\omega)-\lambda| \geq \delta$, for any $\omega \in \Sigma^{*}$.

In [18], Rabin proved that the class of languages recognized by 1pfa's with isolated cut-point coincides with the class of regular languages. A quantum extension of this result is presented in [8] for measure-once automata (MO-1qfa). For more general quantum models, analogous results hold (see, e.g. [5]).

In both probabilistic and quantum interpretations of a formal power series $\varphi$, the stochastic behavior of the automaton imposes the constraint $\operatorname{Im}(\varphi) \subseteq[0,1]$. In analogy to this property, we introduce the more general notion of bounded series: we call $\varphi \in \mathbb{R}\left\langle\left\langle\Sigma^{*}\right\rangle\right\rangle$ a bounded series iff $\sup _{\omega \in \Sigma^{*}}|\varphi(\omega)|<\infty$. Moreover, if $(\pi, \mu, \eta)$ is a linear representation of $\varphi$ and it holds $\sup _{\omega \in \Sigma^{*}}\|\pi \mu(\omega)\|_{2}<\infty$, we say that $(\pi, \mu, \eta)$ is a bounded representation of $\varphi$.

The Hadamard product of two series $\varphi, \psi \in \mathbb{R}\left\langle\left\langle\Sigma^{*}\right\rangle\right\rangle$ is defined as $(\varphi \odot \psi)(\omega)=$ $\varphi(\omega) \cdot \psi(\omega)$. The Hadamard quotient $\frac{\varphi}{\psi}$ is defined as $\frac{\varphi}{\psi}(\omega)=\frac{\varphi(\omega)}{\psi(\omega)}$, whenever $\operatorname{supp}(\psi)=\Sigma^{*}$. It is well-known that the class $\mathbb{R}^{\text {rat }}\left\langle\left\langle\Sigma^{*}\right\rangle\right\rangle$ is closed under Hadamard product, but not under Hadamard quotient, even when $\Sigma$ is a one-letter alphabet. For the unary case, although, the following result has been proved [21]:

Theorem 2.4 (Pisot's conjecture). Let $\sum_{n \in \mathbb{N}} s_{n} z^{n}$ and $\sum_{n \in \mathbb{N}} t_{n} z^{n}$ be two rational power series with coefficients in $\mathbb{R}$. If every $t_{n}$ is different from 0 and $\frac{s_{n}}{t_{n}} \in \mathbb{Z}$ for all $n$, then the power series $\sum_{n \in \mathbb{N}} \frac{s_{n}}{t_{n}} z^{n}$ is rational. 
On a generic finite alphabet, Hadamard quotients of rational series describe the behavior of particular classes of two-way probabilistic automata (2pfa's) [1]. By allowing a two-way motion on the input head, Rabin's theorem does not hold anymore: in [13], Freivalds showed that the nonregular language $\left\{a^{n} b^{n} \mid n \in \mathbb{N}\right\}$ can be recognized by a 2 pfa with isolated cut-point. Dwork and Stockmeyer [12] completed this result showing that if a 2 pfa recognizes a nonregular language with isolated cut-point, then it requires time $2^{n^{b}}$ infinitely often, where $n$ is the length of the input and $b$ is a positive constant. Moreover, this time bound cannot be improved, as shown by Kaneps and Freivalds [15].

\section{Bounded RATIONAL FORMAL POWER SERIES AND ISOlated CUT-POINT}

Fixing a formal power series $\varphi \in \mathbb{R}\left\langle\left\langle\Sigma^{*}\right\rangle\right\rangle$ for a polynomial $P \in \mathbb{R}\left\langle\Sigma^{*}\right\rangle$, the function $\varphi(P)=\sum_{\omega \in \Sigma^{*}} \varphi(\omega) P(\omega)$ is a linear form on $\mathbb{R}\left\langle\Sigma^{*}\right\rangle$. A reduced linear representation of a rational series $\varphi$ of $\mathbb{R}\left\langle\left\langle\Sigma^{*}\right\rangle\right\rangle$ is a linear representation of $\varphi$ with minimal dimension among all its representations.

The following result was proved by Schützenberger in the more general case of rational series over a commutative field (see also [2], Cor. 2.3).

Theorem 3.1 ([20]). If $(\nu, \mu, \eta)$ is a reduced linear representation of dimension $n$ of a rational series $\varphi$ of $\mathbb{R}\left\langle\left\langle\Sigma^{*}\right\rangle\right\rangle$, then there exist polynomials of $\mathbb{R}\left\langle\Sigma^{*}\right\rangle$

$$
P_{1}, \ldots, P_{n}, \quad Q_{1}, \ldots, Q_{n}
$$

such that, for every $\omega \in \Sigma^{*}$ and, for all indexes $i, j \in \mathbb{N}$, with $1 \leq i, j \leq n$

$$
\mu(\omega)_{i j}=\varphi\left(P_{i} \omega Q_{j}\right) .
$$

This allows us to state our main result:

Theorem 3.2. For every reduced linear representation $(\nu, \mu, \eta)$ of a rational series $\varphi \in \mathbb{R}^{\mathrm{rat}}\left\langle\left\langle\Sigma^{*}\right\rangle\right\rangle$, it holds

$$
(\nu, \mu, \eta) \text { is bounded } \Leftrightarrow \varphi \text { is bounded. }
$$

Proof. $(\Rightarrow)$ Since the linear representation is bounded, there exists a positive integer $K$ such that $\|\nu \mu(\omega)\|_{2} \leq K$, for every word $\omega \in \Sigma^{*}$. By applying the Schwarz inequality, for every word $\omega \in \Sigma^{*}$, one thus has

$$
|\varphi(\omega)|=\left|\nu \mu(\omega) \eta^{T}\right| \leq\|\nu \mu(\omega)\|_{2}\|\eta\|_{2} \leq K\|\eta\|_{2} .
$$

$(\Leftarrow)$ Let $(\nu, \mu, \eta)$ be a reduced linear representation of dimension $n$ of a rational series $\varphi$ of $\mathbb{R}\left\langle\left\langle\Sigma^{*}\right\rangle\right\rangle$ such that

$$
|\varphi(\omega)| \leq K
$$


for a fixed positive constant $K$ and for every $\omega \in \Sigma^{*}$. By applying Theorem 3.1 to $(\nu, \mu, \eta)$, there exist $2 n$ polynomials of $\mathbb{R}\left\langle\Sigma^{*}\right\rangle$

$$
P_{1}, \ldots, P_{n}, \quad Q_{1}, \ldots, Q_{n},
$$

such that, for every $\omega \in \Sigma^{*}$ and, for every pair of indexes $i, j \in \mathbb{N}$, with $1 \leq i, j \leq n$,

$$
\mu(\omega)_{i j}=\varphi\left(P_{i} \omega Q_{j}\right) .
$$

For our convenience, let us denote the polynomial $P_{i}(i=1, \ldots, n)$

$$
P_{i}=\sum_{\ell=1}^{\alpha_{i}} c_{i \ell} u_{i \ell}
$$

where $\alpha_{i} \in \mathbb{N}, u_{i \ell} \in \Sigma^{*}$ and $c_{i \ell}=P_{i}\left(u_{i \ell}\right)$ denotes the coefficient associated with $u_{i \ell}$ by $P_{i}$. Similarly, let us denote the polynomial $Q_{j}(j=1, \ldots, n)$

$$
Q_{j}=\sum_{m=1}^{\beta_{j}} d_{j m} v_{j m}
$$

where $\beta_{j} \in \mathbb{N}, v_{j m} \in \Sigma^{*}$ and $d_{j m}=Q_{j}\left(v_{j m}\right)$ denotes the coefficient associated with $v_{j m}$ by $Q_{j}$. Therefore we have

$$
P_{i} \omega Q_{j}=\sum_{\substack{1 \leq \ell \leq \alpha_{i} \\ 1 \leq m \leq \beta_{j}}} c_{i \ell} d_{j m} u_{i \ell} \omega v_{j m}
$$

and hence, by the linearity of $\varphi$ on $\mathbb{R}\left\langle\Sigma^{*}\right\rangle$, we get

$$
\varphi\left(P_{i} \omega Q_{j}\right)=\sum_{\substack{1 \leq \ell \leq \alpha_{i} \\ 1 \leq m \leq \beta_{j}}} c_{i \ell} d_{j m} \varphi\left(u_{i \ell} \omega v_{j m}\right) .
$$

By (3.2)-(3.4), we get

$$
\left|\mu(\omega)_{i j}\right|=\left|\varphi\left(P_{i} \omega Q_{j}\right)\right| \leq \sum_{\substack{1 \leq \ell \leq \alpha_{i}, 1 \leq m \leq \beta_{j}}}\left|c_{i \ell}\right|\left|d_{j m}\right|\left|\varphi\left(u_{i \ell} \omega v_{j m}\right)\right| \leq C_{i j},
$$

where $C_{i j}=K \alpha_{i} \beta_{j} \max _{1 \leq \ell \leq \alpha_{i}}\left|c_{i \ell}\right| \max _{1 \leq m \leq \beta_{j}}\left|d_{j m}\right|$. This implies

$$
\left|\mu(\omega)_{i j}\right| \leq C,
$$

where $C=\max _{1 \leq \ell, m \leq n} C_{\ell m}$. From (2.1) and (3.5), one derives that

$$
\sup _{\omega \in \Sigma^{*}}\|\nu \mu(\omega)\|_{2} \leq \sup _{\omega \in \Sigma^{*}}\left(n\|\nu \mu(\omega)\|_{\infty}\right)=n \sup _{\omega \in \Sigma^{*}}\|\nu \mu(\omega)\|_{\infty} \leq n^{2} C\|\nu\|_{\infty} .
$$

The proof is thus complete. 
The following result is shown in [5]:

Theorem 3.3. Let $\varphi \in \mathbb{R}^{\mathrm{rat}}\left\langle\left\langle\Sigma^{*}\right\rangle\right\rangle$ be a rational formal power series and let $\lambda$ be a real value isolated for $\varphi$. If $\varphi$ has a bounded linear representation, then the language $L_{\varphi, \lambda}$ is regular.

This, together with Theorem 3.2, directly implies

Theorem 3.4. Let $\varphi \in \mathbb{R}^{\text {rat }}\left\langle\left\langle\Sigma^{*}\right\rangle\right\rangle$ be a bounded rational formal power series and let $\lambda$ be a real value isolated for $\varphi$. Then the language $L_{\varphi, \lambda}$ is regular.

\section{Hadamard quotient of RATIONAL SERIES AND REGUlar LANGUAGES}

In the case of a Hadamard quotient of rational formal power series, we cannot directly apply Theorem 3.4 , since $\mathbb{R}^{\text {rat }}\left\langle\left\langle\Sigma^{*}\right\rangle\right\rangle$ is not closed under Hadamard quotient. Instead, it holds

Theorem 4.1. Let $\varphi, \psi \in \mathbb{R}^{\text {rat }}\left\langle\left\langle\Sigma^{*}\right\rangle\right\rangle$ and consider the Hadamard quotient series $\xi=\frac{\varphi}{\psi}$. If

(I) $\xi$ is bounded;

(II) $\psi$ is bounded;

(III) 0 is isolated for $\psi$,

then $L_{\xi, \lambda}$ is a regular language for every $\lambda$ that is isolated for $\xi$.

Proof. The facts that $\psi$ and $\xi$ are bounded imply that also $\varphi$ is bounded. Indeed, if $|\psi(\omega)| \leq M_{1}$ and $\left|\frac{\varphi(\omega)}{\psi(\omega)}\right| \leq M_{2}$, then $|\varphi(\omega)| \leq M_{2}|\psi(\omega)| \leq M_{1} M_{2}$. Since 0 is isolated for $\psi$, there exists an $\varepsilon>0$ such that $(\psi(\omega))^{2} \geq \varepsilon$, and since $\lambda$ is isolated for $\xi$, there exists a $\delta>0$ such that $\left|\frac{\varphi(\omega)}{\psi(\omega)}-\lambda\right| \geq \delta$.

The language in which we are interested is

$$
L_{\xi, \lambda}=\left\{\omega \in \Sigma^{*} \mid \frac{\varphi(\omega)}{\psi(\omega)}>\lambda\right\}=\left\{\omega \in \Sigma^{*} \mid \varphi(\omega) \psi(\omega)-\lambda(\psi(\omega))^{2}>0\right\} .
$$

It holds

$$
\begin{aligned}
\left|\frac{\varphi(\omega)}{\psi(\omega)}-\lambda\right| \geq \delta & \Leftrightarrow\left|\varphi(\omega) \psi(\omega)-\lambda(\psi(\omega))^{2}\right| \geq \delta(\psi(\omega))^{2} \\
& \Rightarrow\left|\varphi(\omega) \psi(\omega)-\lambda(\psi(\omega))^{2}\right| \geq \delta \varepsilon
\end{aligned}
$$

which proves that 0 is isolated for $\varphi(\omega) \psi(\omega)-\lambda(\psi(\omega))^{2}$. Moreover,

$$
\left|\varphi(\omega) \psi(\omega)-\lambda(\psi(\omega))^{2}\right| \leq|\varphi(\omega)| \cdot|\psi(\omega)|+|\lambda| \cdot(\psi(\omega))^{2} \leq M_{1}^{2}\left(M_{2}+\lambda\right)
$$

is bounded, so, by Theorem $3.4, L_{\xi, \lambda}$ is a regular language. 
In what follows, we will show that, if one of conditions (I)-(III) is dropped, then Theorem 4.1 does not hold anymore, even in the unary case. Our first main result is

Theorem 4.2. Let $\varphi\left(\sigma^{n}\right)=n^{2} \sin ^{2}(\pi n \theta)$, where $\theta=\frac{1+\sqrt{5}}{2}$. For every rational $\rho>\frac{\pi^{2}}{5}$, there exists a rational $\lambda \geq \rho$ such that:

- $L_{\varphi, \rho}$ is not context-free;

- $\lambda$ is isolated for $\varphi$ and $L_{\varphi, \lambda}=L_{\varphi, \rho}$.

As a direct consequence of the previous result, we obtain

Theorem 4.3. If one of conditions (I)-(III) in Theorem 4.1 is dropped, then there exists an isolated $\lambda$ for $\xi$ such that the language $L_{\xi, \lambda}$ is not context free.

Proof. In this proof, we will show three Hadamard quotients of formal power series in $\mathbb{R}^{\text {rat }}\left\langle\left\langle\{\sigma\}^{*}\right\rangle\right\rangle$, satisfying only two of the conditions in Theorem 4.1 and characterizing, with an isolated cut point, the non-context-free language $L_{\varphi, \lambda}$ defined in Theorem 4.2.

By dropping condition (I) we consider the power series $\xi_{1}=\frac{\varphi_{1}\left(\sigma^{n}\right)}{\psi_{1}\left(\sigma^{n}\right)}$, where $\varphi_{1}\left(\sigma^{n}\right)=n^{2} \sin ^{2}(\pi n \theta)$ is rational and unbounded, while $\psi_{1}\left(\sigma^{n}\right)=1$ respects conditions (II) and (III). Clearly, $L_{\xi_{1}, \lambda}=\left\{\sigma^{n} \mid \xi_{1}\left(\sigma^{n}\right)>\lambda\right\}=L_{\varphi, \lambda}$.

By dropping condition (II), we consider the series $\xi_{2}=\frac{\varphi_{2}}{\psi_{2}}$, where $\varphi_{2}\left(\sigma^{n}\right)=$ $n^{2} \sin ^{2}(\pi n \theta)+1$ and $\psi_{2}\left(\sigma^{n}\right)=n^{2} \sin ^{2}(\pi n \theta)+2$, so that $\psi_{2}\left(\sigma^{n}\right) \geq 2$ and $\xi_{2}\left(\sigma^{n}\right) \leq 1$ for all $n$. By considering the function $f(x)=\frac{x+1}{x+2}$, we can write $\xi_{2}\left(\sigma^{n}\right)=f\left(\xi_{1}\left(\sigma^{n}\right)\right)$. We are going to show that $f(\lambda)$ is isolated for $\xi_{2}$ : indeed, since $f$ is nondecreasing, whenever $\xi_{1}\left(\sigma^{n}\right) \geq \lambda+\varepsilon$, for some $0<\varepsilon \leq \lambda$, it holds $\xi_{2}\left(\sigma^{n}\right) \geq f(\lambda+\varepsilon)=$ $f(\lambda)+\frac{\varepsilon}{(\lambda+\varepsilon+2)(\lambda+2)}$. Symmetrically, whenever $\xi_{1}\left(\sigma^{n}\right) \leq \lambda-\varepsilon$, it holds $\xi_{2}\left(\sigma^{n}\right) \leq$ $f(\lambda-\varepsilon)=f(\lambda)-\frac{\varepsilon}{(\lambda-\varepsilon+2)(\lambda+2)}$, so the claim is settled, and it holds

$$
L_{\xi_{2}, \frac{\lambda+1}{\lambda+2}}=\left\{\sigma^{n} \mid \xi_{2}\left(\sigma^{n}\right)>f(\lambda)\right\}=\left\{\sigma^{n} \mid \xi_{1}\left(\sigma^{n}\right)>\lambda\right\}=L_{\varphi, \lambda} .
$$

By dropping condition (III), we consider the series $\xi_{3}=\frac{\varphi_{3}}{\psi_{3}}$, where $\varphi_{3}\left(\sigma^{n}\right)=$ $2^{-n}\left(n^{2} \sin ^{2}(\pi n \theta)+1\right)$ and $\psi_{3}\left(\sigma^{n}\right)=2^{-n}\left(n^{2} \sin ^{2}(\pi n \theta)+2\right)$ are both bounded, but $\psi_{3}$ approaches zero as $n$ grows. Clearly, $\xi_{3}=\xi_{2}$, so $f(\lambda)$ is isolated for $\xi_{3}$ and

$$
L_{\xi_{3}, \frac{\lambda+1}{\lambda+2}}=\left\{\sigma^{n} \mid \xi_{3}\left(\sigma^{n}\right)>f(\lambda)\right\}=L_{\xi_{2}, f(\lambda)}=L_{\varphi, \lambda}
$$

Now, we prove Theorem 4.2: let $\theta_{1}=\frac{1+\sqrt{5}}{2}$ and $\theta_{2}=\frac{1-\sqrt{5}}{2}$ be the positive and negative roots of the equation $x^{2}-x-1=0$. Our aim is to show that the limit points of the sequence

$$
\phi(n)=\left|n \sin \left(\pi n \theta_{1}\right)\right|
$$

are of type $\left|b^{2}-a b-a^{2}\right| \frac{\pi}{\sqrt{5}}$, with $a, b$ integers and $b>a \geq 0$.

Lemma 4.4. If $z$ is a limit point of $\phi$ then there exist integers $b>a \geq 0$ such that $z=\frac{\left|b^{2}-a b-a^{2}\right| \pi}{\sqrt{5}}$. 
Proof. Let $z \geq 0$ be a limit point of the sequence $\phi$. There exists a subsequence $\left|n_{k} \sin \left(\pi n_{k} \theta_{1}\right)\right|$ such that

$$
\lim _{k \rightarrow \infty}\left|n_{k} \sin \left(\pi n_{k} \theta_{1}\right)\right|=z
$$

Since $\left|\sin \left(\pi n_{k} \theta_{1}\right)\right|=\left|\sin \left(\pi\left\{n_{k} \theta_{1}\right\}\right)\right|$, it holds: $\lim _{k \rightarrow \infty}\left|n_{k} \sin \left(\pi\left\{n_{k} \theta_{1}\right\}\right)\right|=z$, and this implies:

$$
\lim _{k \rightarrow \infty}\left|\sin \left(\pi\left\{n_{k} \theta_{1}\right\}\right)\right|=\lim _{k \rightarrow \infty} \frac{z}{n_{k}}=0 .
$$

Moreover, since $\left\{n_{k} \theta_{1}\right\} \leq \frac{1}{2}$, by Lemma 2.1 we have:

$$
\left|\sin \left(\pi\left\{n_{k} \theta_{1}\right\}\right)\right| \sim \pi\left\{n_{k} \theta_{1}\right\} .
$$

Equations (4.2)-(4.4) imply the following:

$$
\lim _{k \rightarrow \infty} n_{k}\left\{n_{k} \theta_{1}\right\}=\frac{z}{\pi},
$$

and

$$
\lim _{k \rightarrow \infty}\left\{n_{k} \theta_{1}\right\}=0
$$

By the definition of the quantization error, for every $n_{k}$, there exists an integer $m_{k}$ such that:

$$
\left\{n_{k} \theta_{1}\right\}=\left|m_{k}-n_{k} \theta_{1}\right|
$$

so that

$$
\lim _{k \rightarrow \infty}\left|\frac{m_{k}}{n_{k}}-\theta_{1}\right|=\lim _{k \rightarrow \infty} \frac{\left|m_{k}-n_{k} \theta_{1}\right|}{n_{k}}=\lim _{k \rightarrow \infty} \frac{\left\{n_{k} \theta_{1}\right\}}{n_{k}}=0,
$$

and that implies:

$$
\frac{m_{k}}{n_{k}} \sim \theta_{1}
$$

The numbers $\theta_{1}$ and $\theta_{2}$ satisfy the equality:

$$
\left|\frac{m_{k}}{n_{k}}-\theta_{1}\right|\left|\frac{m_{k}}{n_{k}}-\theta_{2}\right|=\frac{\left|m_{k}^{2}-m_{k} n_{k}-n_{k}^{2}\right|}{n_{k}^{2}} .
$$

By the latter together with (4.5) and (4.7), we get:

$$
\begin{aligned}
\lim _{k \rightarrow \infty}\left|m_{k}^{2}-m_{k} n_{k}-n_{k}^{2}\right| & =\lim _{k \rightarrow \infty} n_{k}\left|m_{k}-n_{k} \theta_{1}\right|\left|\frac{m_{k}}{n_{k}}-\theta_{2}\right| \\
& =\left(\lim _{k \rightarrow \infty} n_{k}\left\{n_{k} \theta_{1}\right\}\right)\left(\lim _{k \rightarrow \infty}\left|\frac{m_{k}}{n_{k}}-\theta_{2}\right|\right) \\
& =\frac{z}{\pi}\left|\theta_{1}-\theta_{2}\right|=\frac{z \sqrt{5}}{\pi} .
\end{aligned}
$$

Since $\left|m_{k}^{2}-m_{k} n_{k}-n_{k}^{2}\right|$ is a convergent sequence of integers, there exists an integer $c$ such that, for a sufficiently large $\bar{k}$, it holds for every $k \geq \bar{k}$ : $\mid m_{k}^{2}-$ $m_{k} n_{k}-n_{k}^{2} \mid=c$. Since $c=\frac{z \sqrt{5}}{\pi}$, we have $z=\frac{c \pi}{\sqrt{5}}$. 
Moreover, since $\frac{m_{k}}{n_{k}} \sim \theta_{1}>1$ we can assume $\bar{k}$ big enough so that it holds $m_{\bar{k}}>n_{\bar{k}}$. By setting $b=m_{\bar{k}}$ and $a=n_{\bar{k}}$, we obtain: $b>a \geq 0$ and $z=\frac{\left|b^{2}-a b-a^{2}\right| \pi}{\sqrt{5}}$. This concludes the proof.

Lemma 4.5. Let $a, b$ be integers such that $b>a \geq 0$ and $z=\frac{\left|b^{2}-a b-a^{2}\right| \pi}{\sqrt{5}}$. Then $z$ is a limit point of $\phi$.

Proof. We consider the sequence $F_{k}$ wich is the solution of the recurrence equation

$$
u_{k+2}=u_{k+1}+u_{k}
$$

with the initial conditions $F_{0}=a$ and $F_{1}=b$. Elementary computations show that

$$
F_{k}=\alpha\left(\theta_{1}\right)^{k}+\beta\left(\theta_{2}\right)^{k}
$$

where $\alpha=\frac{b-a \theta_{2}}{\sqrt{5}}$ and $\beta=\frac{a \theta_{1}-b}{\sqrt{5}}$. In particular, since $\left|\theta_{2}\right|<1$, we have $F_{k} \sim \alpha \theta_{1}^{k}$.

This yiels $\theta_{1} F_{k}-F_{k+1}=\beta\left(\theta_{1}-\theta_{2}\right) \theta_{2}^{k}=\beta \sqrt{5} \theta_{2}^{k}$; since $\lim _{k \rightarrow \infty} \theta_{2}^{k}=0, F_{k+1}$ is the best integer approximation of $\theta_{1} F_{k}$. Hence, for sufficiently large $k$, we get

$$
\left\{\theta_{1} F_{k}\right\}=\left|\beta \sqrt{5} \theta_{2}^{k}\right|
$$

As consequence:

$$
F_{k}\left\{\theta_{1} F_{k}\right\}=F_{k}\left|\beta \sqrt{5} \theta_{2}^{k}\right| \sim\left|\sqrt{5} \alpha \beta\left(\theta_{1} \theta_{2}\right)^{k}\right|=\frac{\left|b^{2}-a b-a^{2}\right|}{\sqrt{5}},
$$

since $\theta_{1} \theta_{2}=-1$ and $\alpha \beta=\frac{-\left(b^{2}-a b-a^{2}\right)}{5}$. In conclusion, we have:

$$
\left|F_{k} \sin \left(\pi F_{k} \theta_{1}\right)\right|=\left|F_{k} \sin \left(\pi\left\{\theta_{1} F_{k}\right\}\right)\right| \sim F_{k} \pi\left\{\theta_{1} F_{k}\right\} \sim \frac{\pi\left|b^{2}-a b-a^{2}\right|}{\sqrt{5}} .
$$

As an immediate consequence of the previous two lemmas we get the following characterization of the limit points of $\phi[9]$ :

\section{Lemma 4.6.}

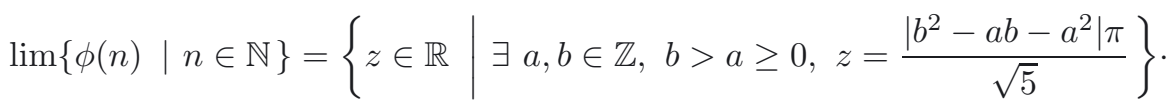

Now we are ready to prove Theorem 4.2.

Proof of Theorem 4.2. We recall that

$$
\varphi\left(\sigma^{n}\right)=\phi^{2}(n)=n^{2} \sin ^{2}(\pi n \theta),
$$


therefore, by Lemma 4.6, the limit points of $\varphi$ are of the form $\frac{\left(b^{2}-a b-a^{2}\right)^{2} \pi^{2}}{5}$ for some integers $b>a \geq 0$ and, in particular, $\frac{\pi^{2}}{5}$ is a limit point. Since any unary context-free language is also regular, our main task amounts to show that

$$
L_{\varphi, \rho}^{c}=L=\left\{\sigma^{n} \mid \varphi\left(\sigma^{n}\right) \leq \rho\right\}
$$

is not regular. By letting $F_{k}$ be the Fibonacci sequence, which can be obtained by the recurrence equation (4.8) by setting $a=0$ and $b=1$, Equation (4.9) translates into $\lim _{k \rightarrow \infty} F_{k}\left(\pi\left\{F_{k} \theta\right\}\right)=\frac{\pi}{\sqrt{5}}$, therefore

$$
\lim _{k \rightarrow \infty} \varphi\left(F_{k}\right)=\lim _{k \rightarrow \infty} F_{k}^{2} \sin ^{2}\left(F_{k} \pi \theta\right)=\lim _{k \rightarrow \infty} F_{k}^{2}\left(\pi\left\{F_{k} \theta\right\}\right)^{2}=\frac{\pi^{2}}{5},
$$

so there exist infinitely many values $k$ such that $a^{F_{k}} \in L$, since we assumed $\rho>\frac{\pi^{2}}{5}$.

Suppose, by contradiction, that $L$ is regular. Then, because of the Pumping Lemma, there exist integers $h>0$ and $d>0$ such that $a^{F_{h}+\gamma d} \in L$, for all $\gamma \in \mathbb{N}$, which translates into the following condition

$$
\left(F_{h}+\gamma d\right)^{2} \cdot \sin ^{2}\left(\pi\left(F_{h}+\gamma d\right) \theta\right) \leq \rho
$$

The defining condition of $L$ is $\sin ^{2}(\pi n \theta) \leq \frac{\rho}{n^{2}}$ and, thus, for $\gamma \rightarrow \infty$ it holds $\sin ^{2}\left(\pi\left(F_{h}+\gamma d\right) \theta\right) \sim\left(\left\{\pi F_{h} \theta+\gamma \pi d \theta\right\}\right)^{2}$. However, since $\theta$ is irrational, the set $\left\{\left\{\pi F_{h} \theta+\gamma \pi d \theta\right\} \mid \gamma \in \mathbb{N}\right\}$ is dense in $\left[0, \frac{1}{2}\right]$, so for every constant $\tau \in\left[0, \frac{1}{2}\right]$ there exists a subsequence $\gamma_{1}<\gamma_{2}<\ldots<\gamma_{t}<\ldots$ such that

$$
\lim _{t \rightarrow \infty}\left(\left\{\pi F_{h} \theta+\gamma_{t} \pi d \theta\right\}\right)^{2}=\tau^{2}
$$

which implies that $\left(F_{h}+\gamma_{t} d\right)^{2} \cdot \sin ^{2}\left(\pi\left(F_{h}+\gamma_{t} d\right) \theta\right)$ diverges, against (4.10).

In order to prove the second statement of the theorem, it is sufficient to notice that $\rho$ is not a limit point of $\varphi$. Therefore, we can always find a rational value $\lambda \geq \rho$ isolated for $\varphi$ and close enough to $\rho$, so that it holds $L_{\varphi, \lambda}=L_{\varphi, \rho}$.

\section{DeCision PROBlems ON RATIONAL POWER SERIES}

In the previous sections we have analyzed the languages defined by formal power series with isolated cut point and took into consideration some properties of those series in order to guarantee the regularity of the associated language. Now we investigate the decidability of these properties. In the literature there are many undecidability results on formal power series and, in particular, on 1pfa's. A crucial problem on 1pfa's is the isolation problem: given a $1 \mathrm{pfa} A$ and a cut point $\lambda$, do there exist words whose acceptance probability is arbitrarily close to $\lambda$ ? This problem has been shown to be undecidable even for values of $\lambda$ in the interval 
$(0,1)[3,4]$ (see $[16]$ for a different proof). The result may be written in terms of rational series as follows:

Lemma 5.1. [3,4] Given a rational series $\varphi \in \mathbb{R}^{\text {rat }}\left\langle\left\langle\Sigma^{*}\right\rangle\right\rangle$, the problem of determining whether $\frac{1}{2}$ is isolated for $\varphi$ is undecidable.

The problem remains undecidable for rational series with reduced representation of fixed dimension [6]. Notice that, however, if we restrict ourselves to series where the matrices in the linear representation belong to a compact group (e.g. unitary matrices), then the isolation problem becomes decidable, as shown in [7] for quantum automata.

Another important decidability result on power series is stated in [19]:

Lemma 5.2. Given an alphabet $\Sigma$ such that $|\Sigma| \geq 2$ and a rational series with integer coefficients $\varphi \in \mathbb{Z}^{\text {rat }}\left\langle\left\langle\Sigma^{*}\right\rangle\right\rangle$, it is undecidable whether there exists a word $\omega \in \Sigma^{*}$ such that $\varphi(\omega)=0$.

The first decision problem we approach concerns the regularity of the language defined by a rational series with isolated cut point. Notice that, as stated in Lemma 5.1, the isolation problem itself is undecidable, so the set of all the rational series with isolated cut point is non-recursive. However, we show that there exists a recursive subclass of rational power series with isolated cut point such that the regularity of the associated language is undecidable.

Theorem 5.3. There exists a recursive set $I$ of rational series $\varphi \in \mathbb{Q}^{\text {rat }}\left\langle\left\langle\Sigma^{*}\right\rangle\right\rangle$ such that the value $\lambda=\frac{2}{3}$ is isolated for every series $\varphi \in I$, and such that the problem of deciding whether the language $L_{\varphi, \lambda}$ is regular is undecidable.

Proof. We first remark that Lemma 5.2 still holds in the case of alphabets with only two symbols. Therefore, in the sequel, we restrict ourselves to consider a rational series with integer coefficients $\psi \in \mathbb{Z}^{\text {rat }}\left\langle\left\langle\{a, b\}^{*}\right\rangle\right\rangle$ on the alphabet $\{a, b\}$. Let us define a new rational series $\varphi \in \mathbb{Q}^{\text {rat }}\left\langle\left\langle\{a, b, c, d\}^{*}\right\rangle\right\rangle$ as follows:

$$
\varphi(\omega)=2^{\# c(\omega)-\#_{d}(\omega)}+\psi^{2}\left(P_{\{a, b\}}(\omega)\right),
$$

where $P_{\{a, b\}}(\omega)$ is the projection of $\omega$ on the alphabet $\{a, b\}$. Since $\varphi$ is of the form $2^{\alpha}+\beta$, with $\alpha \in \mathbb{Z}$ and $\beta \in \mathbb{N}$, the value $\lambda=\frac{2}{3}$ is isolated for $\varphi$. In order to prove the undecidability of our problem, we are going to show that $L_{\varphi, \lambda}$ is regular iff there exists no word $x \in\{a, b\}^{*}$ such that $\psi(x)=0$. We first analyze the case where there is no such word $x$ and, since $\psi^{2}$ only assumes values in $\mathbb{N}$, this implies that $\psi^{2}(x) \geq 1$ for all $x \in\{a, b\}^{*}$. Then, for all $\omega \in\{a, b, c, d\}$ it holds $\varphi(\omega)=2^{\#_{c}(\omega)-\#_{d}(\omega)}+1 \geq 1>\lambda$, so the language $L_{\varphi, \lambda}=\{a, b, c, d\}^{*}$ is regular. Instead, if there exists a word $\bar{x}$ such that $\psi(\bar{x})=0$, for each word $\omega \in L=(c+d)^{*} \bar{x}$ it holds $\varphi(\omega)=2^{\# c(\omega)-\#_{d}(\omega)}+0$, and therefore

$$
\varphi(\omega)>\frac{2}{3} \Leftrightarrow \#_{c}(\omega) \geq \#_{d}(\omega)
$$


so the language $L_{\varphi, \lambda} \cap L$ is not regular, and since $L$ is regular, $L_{\varphi, \lambda}$ must be not regular.

The second decision problem we consider concerns one of the regularity conditions we gave in Corollary 3.4:

Theorem 5.4. Given a rational formal power series $\varphi \in \mathbb{R}^{\mathrm{rat}}\left\langle\left\langle\Sigma^{*}\right\rangle\right\rangle$, the problem of determining whether $\varphi$ is bounded is undecidable.

Proof. Consider a rational series $\zeta \in \mathbb{R}^{\text {rat }}\left\langle\left\langle\Sigma^{*}\right\rangle\right\rangle$. Because of Schützenberger's theorem and because of the closure properties of $\mathbb{R}^{\text {rat }}\left\langle\left\langle\Sigma^{*}\right\rangle\right\rangle$, we can always define a linear representation $A=(\nu, \mu, \eta)$ such that $\phi(\omega)=\nu \mu(\omega) \eta^{T}=1-(2 \zeta(\omega)-1)^{2}$. Without loss of generality we can assume $\Sigma=\{a, b\}$, and it still holds, for Lemma 5.1, that asking whether $\frac{1}{2}$ is isolated for $\zeta$ is undecidable, so also the problem of determining whether 1 is isolated for $\phi$ is undecidable. We want to find a rational series which is bounded if and only if 1 is isolated for $\phi$. By calling $m$ the dimension of $A$, we define a new linear representation $\hat{A}=(\hat{\nu}, \hat{\mu}, \hat{\eta})$ of dimension $m+1$ on the alphabet $\hat{\Sigma}=\{a, b, c, d\}$ such that:

- $\hat{\nu}=e_{2}=(0,1,0, \ldots, 0) \in \mathbb{R}^{1 \times(m+1)}$;

- $\hat{\eta}=e_{1}=(1,0, \ldots, 0) \in \mathbb{R}^{1 \times(m+1)}$;

- $\hat{\mu}(a)=(1) \oplus \mu(a)$

- $\hat{\mu}(b)=(1) \oplus \mu(b)$;

- $\hat{\mu}(c)$ is the matrix where the only nonzero components are the first two rows $r_{1}=e_{1}$ and $r_{2}=(1) \oplus \nu$;

- $\hat{\mu}(d)$ is the matrix where the only nonzero components are the first two columns $c_{1}=e_{1}^{T}$ and $c_{2}=((0) \oplus \eta)^{T}$.

For any $x \in\{a, b\}^{*}$ we have the following

$$
\begin{aligned}
& \hat{\mu}(c x d)=\left(\begin{array}{cc}
1 & \mathbf{0}^{1 \times m} \\
1 & \mu \\
& \\
\mathbf{0}^{(m-1) \times(m+1)}
\end{array}\right)\left(\begin{array}{cc}
1 & \mathbf{0}^{1 \times m} \\
\mathbf{0}^{m \times 1} & \mu(x)
\end{array}\right)\left(\begin{array}{ccc}
1 & 0 & \mathbf{0}^{(m+1) \times(m-1)} \\
\mathbf{0}^{m \times 1} & \eta &
\end{array}\right. \\
& =\left(\begin{array}{ccc}
1 & 0 & \mathbf{0}^{2 \times(m-1)} \\
1 & \phi(x) & \\
\mathbf{0}^{(m-1) \times 2} & \mathbf{0}^{(m-1) \times(m-1)}
\end{array}\right) .
\end{aligned}
$$

For simplicity, we can reduce the above matrix to the top left block containing the only nonzero values when $\mu$ is applied to words in the language $L=(c(a+$ $\left.b)^{*} d\right)^{*}$, so the coefficients of the series $\psi(\omega)=\hat{\nu} \hat{\mu}(\omega) \hat{\eta}^{T}$ can be obtained as follows

$$
\psi\left(c x_{1} d c x_{2} d \cdots c x_{s} d\right)=\left(\begin{array}{ll}
0 & 1
\end{array}\right)\left(\begin{array}{cc}
1 & 0 \\
1 & \phi\left(x_{1}\right)
\end{array}\right) \cdots\left(\begin{array}{cc}
1 & 0 \\
1 & \phi\left(x_{s}\right)
\end{array}\right)\left(\begin{array}{l}
1 \\
0
\end{array}\right)
$$

where $x_{1}, x_{2}, \ldots, x_{s} \in\{a, b\}^{*}$. The rational series we consider to prove the theorem is $\varphi=\chi_{L} \odot \psi$ : we are going to show that $\varphi$ is bounded iff 1 is isolated for $\phi$. Let 
us start with the case where 1 is isolated for $\phi$, which means there exists a value $\rho<1$ such that $\phi(x) \leq \rho$ for all $x \in\{a, b\}^{*}$, so, for any $\omega=c x_{1} d c x_{2} d \cdots c x_{s} d \in L$, we have that

$$
\psi(\omega) \leq\left(\begin{array}{ll}
0 & 1
\end{array}\right)\left(\begin{array}{ll}
1 & 0 \\
1 & \rho
\end{array}\right)^{s}\left(\begin{array}{l}
1 \\
0
\end{array}\right)=\sum_{j=0}^{s-1} \rho^{j}=\frac{1-\rho^{s}}{1-\rho}<\frac{1}{1-\rho}
$$

is bounded. In the case 1 is not isolated for $\phi$, there exists a sequence $S=\left\{x_{n} \mid x_{n} \in\right.$ $\left.\{a, b\}^{*}, n \in \mathbb{N}\right\}$ such that $\lim _{n \rightarrow \infty} \phi\left(x_{n}\right)=1$. We choose the sequence $S$ such that $\phi\left(x_{n}\right)>1-\frac{1}{n}$, and we consider the sequence of words $\left\{\left(c x_{n} d\right)^{n} \mid x_{n} \in S, n \in \mathbb{N}\right\}$. It holds

$$
\psi\left(\left(c x_{n} d\right)^{n}\right)=\sum_{j=0}^{n-1} \phi^{j}\left(x_{n}\right)=\frac{1-\phi^{n}\left(x_{n}\right)}{1-\phi\left(x_{n}\right)} .
$$

Since $\frac{1-x^{n}}{1-x}$ is a monotonic increasing function, we have

$$
\psi\left(\left(c x_{n} d\right)^{n}\right) \geq \frac{1-\left(1-\frac{1}{n}\right)^{n}}{\frac{1}{n}} \sim\left(1-e^{-1}\right) n
$$

which diverges for $n \rightarrow \infty$, so the series $\varphi=\chi_{L} \odot \psi$ is not bounded.

It is worth noticing that the boundedness problem is decidable in $\mathbb{Z}^{\text {rat }}\left\langle\left\langle\Sigma^{*}\right\rangle\right\rangle$. Indeed in this case the question is equivalent to asking whether the series have a finite image, which is a decidable problem [14].

\section{REFERENCES}

[1] M. Anselmo and A. Bertoni, On 2pfas and the Hadamard quotient of formal power series. Bull. Belg. Math. Soc. 1 (1994) 165-173.

[2] J. Berstel and C. Reutenauer, Rational Series and Their Languages. Springer-Verlag (1988).

[3] A. Bertoni, The solution of problems relative to probabilistic automata in the frame of the formal languages theory, in Vierte Jahrestagung der Gesellschaft for Informatik. Lect. Notes Comput. Sci. 26 (1975) 107-112.

[4] A. Bertoni, G. Mauri and M. Torelli, Some recursively unsolvable problems relating to isolated cutpoints in probabilistic automata, in Proc. of 4th International Colloquium on Automata, Languages and Programming. Lect. Notes Comput. Sci. 52 (1977) 87-94.

[5] A. Bertoni, C. Mereghetti and B. Palano, Quantum Computing: 1-Way Quantum Automata, in Proc. of Developments in Language Theory. Lect. Notes Comput. Sci. (2003) 1-20.

[6] V.D. Blondel and V. Canterini, Undecidable problems for probabilistic automata of fixed dimension. Theor. Comput. Syst. 36 (2003) 231-245.

[7] V.D. Blondel, E. Jeandel, P. Koiran and N. Portier, Decidable and undecidable problems about quantum automata. SIAM J. Comput. 34 (2005) 1464-1473

[8] A. Brodsky and N. Pippenger, Characterization of 1-way quantum finite automata. Available on http://xxx.lanl.gov/abs/quant-ph/9903014.

[9] C. Choffrut, Private communication to the authors, July 2011.

[10] N. Chomsky and M.P. Schützenberger, The Algebraic Theory of Context-Free Languages, in Computer Programming and Formal Systems. North-Holland, Amsterdam (1963).

[11] M. Droste, W. Kuich and H. Vogler, Handbook of weighted automata. EATCS Series Springer (2009). 
[12] C. Dwork and L. Stockmeyer, A time complexity gap for two-way probabilistic finite-state automata. SIAM J. Comput. 19 (1990) 1011-1023.

[13] R. Freivalds, Probabilistic Two-way Machines, in Proc. of Int. Symp. Math. Found. Comput. Sci. Lect. Notes Comput. Sci. 118 (1981) 33-45.

[14] G. Jacob, La finitude des représentations linéaires des semigoupes est décidable. J. Algebra 52 (1978) 437-459.

[15] J. Kaneps and R. Freivalds, Running time to recognize nonregular languages by two-way probabilistic automata, in Proc. of ICALP 91. Lect. Notes Comput. Sci. 510 (1991) 174185.

[16] O. Madani, S. Hanks and A. Condon, On the undecidability of probabilistic planning and infinite-horizon partially observable markov decision problems, in Proc. of the 6th National Conference on Artificial Intelligence (1999).

[17] A. Paz, Introduction to Probabilistic Automata. Academic Press (1971).

[18] M. Rabin, Probabilistic automata. Inf. Control 6 (1963) 230-245.

[19] A. Salomaa and M. Soittola, Automata-theoretic aspects of formal power series. Springer (1978).

[20] M.P. Schützenberger, On the definition of a family of automata. Inf. Control 4 (1961) 245270.

[21] A.J. Van Der Poorten, Solution de la conjecture de Pisot sur le quotient de Hadamard de deux fractions rationnelles. C. R. Acad. Sci. Paris, Sér. I 306 (1988) 97-102.

Communicated by R. Freund.

Received November 11, 2011. Accepted June 8, 2012. 